Case Report

\title{
Chronic Intussusception Associated with Malrotation in a Child: A Variation of Waugh's Syndrome?
}

\author{
Nick Zavras, ${ }^{1}$ Konstantinos Tsilikas, ${ }^{2}$ and George $\operatorname{Vaos}^{1}$ \\ ${ }^{1}$ Department of Paediatric Surgery, "ATTIKON" University General Hospital, School of Medicine, \\ National and Kapodistrian University of Athens, Athens, Greece \\ ${ }^{2}$ Department of Radiology, Penteli General Children's Hospital, Athens, Greece
}

Correspondence should be addressed to George Vaos; gvaos@med.uoa.gr

Received 17 July 2016; Accepted 14 September 2016

Academic Editor: Francis A. Abantanga

Copyright (C) 2016 Nick Zavras et al. This is an open access article distributed under the Creative Commons Attribution License, which permits unrestricted use, distribution, and reproduction in any medium, provided the original work is properly cited.

\begin{abstract}
Chronic intussusception is a relatively uncommon disease most commonly observed in older children. Waugh's syndrome represents a rare entity characterized by intestinal malrotation and acute intussusception. We report a very unusual case of intestinal malrotation associated with chronic intussusception. Clinical presentation, radiological findings, and managing of this association are discussed in the light of the available literature.
\end{abstract}

\section{Introduction}

Acute intussusception is a common emergency abdominal condition in children [1]. Common clinical patterns include intermittent abdominal pain, vomiting, and "currant jelly" bloody stool [2]. Intestinal malrotation is a congenital condition caused by abnormal rotation and fixation of the bowel [3]. While bilious vomiting is the most frequent symptom in neonates, in older children intestinal malrotation is most commonly associated with nonspecific symptoms, such as chronic abdominal pain, malabsorption, diarrhea, or constipation, which may delay diagnosis [4]. The association of acute intussusception with intestinal malrotation is known as Waugh's syndrome (WS) [5]. Chronic intussusception is a distinct clinical entity, characterized by intermittent attacks of abdominal pain lasting more than 14 days; other symptoms of acute intussusception may not present. One impressive clinical feature is significant weight loss due to long-standing anorexia and vomiting [6].

Herein, we describe a rare case of chronic intussusception with intestinal malrotation that could possibly represent a variation of WS. A brief literature review of paediatric cases with WS is presented.

\section{Case Report}

A previously healthy 4.5 -year-old boy was admitted to our department with a 6-week history of intermittent abdominal pain, poor appetite, sporadic nonbilious vomiting, and occasional constipation. A weight loss of five kilograms since the onset of symptoms was reported. On admission, the weight of the patient was $15 \mathrm{~kg}$, as opposed to $20 \mathrm{~kg}$ six weeks earlier. Physical examination revealed a soft and mildly distended abdomen. A palpable, tender, round mobile mass was detected at the epigastrium. The white blood cell count was $11.000 / \mu \mathrm{L}$ (normal range, $4.500-9.900 / \mu \mathrm{L}$ ), hemoglobin was $12.1 \mathrm{~g} / \mathrm{dL}$, and platelets were $420.000 / \mu \mathrm{L}$. The serum chemistry profile was within normal limits, apart from C-reactive protein of $0.8 \mathrm{mg} / \mathrm{mL}$ (normal range, 0$0.5 \mathrm{mg} / \mathrm{mL}$ ). Abdominal ultrasonography in transverse view revealed alternating hypoechoic and hyperechoic bowel walls suggesting the target sign (Figure 1). Hydrostatic reduction was attempted, without success (Figure 2).

Exploratory laparotomy through a right upper quadrant transverse incision revealed an ileocolic intussusception extending up to the transverse colon (Figure 3(a)). The duodenojejunal junction was found to be on the right of 


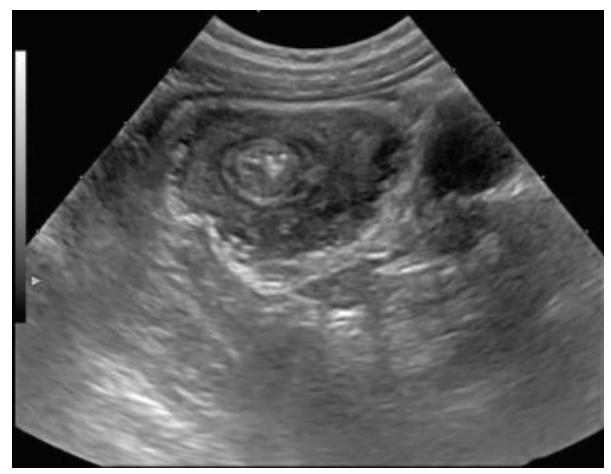

FIgURE 1: Axial U/S view of ileocolic intussusception: multiple concentric ring/donut sign.

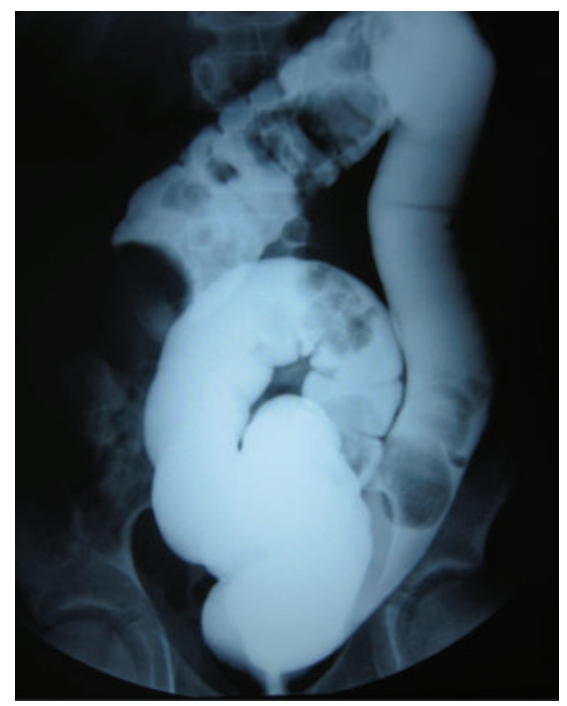

FIGURE 2: Barium contrast enema: configuration of the unsuccessfully reduced intussusception in the right upper quadrant.

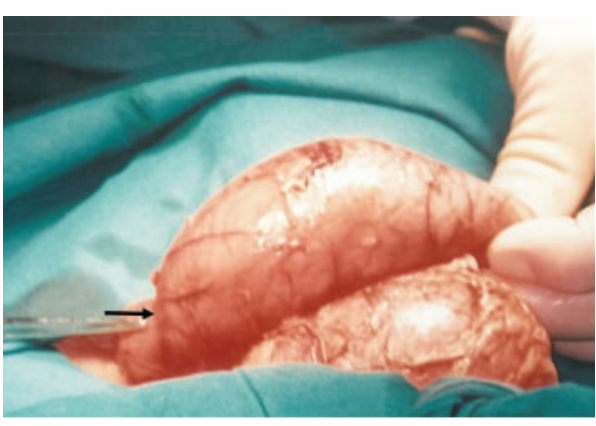

(a)

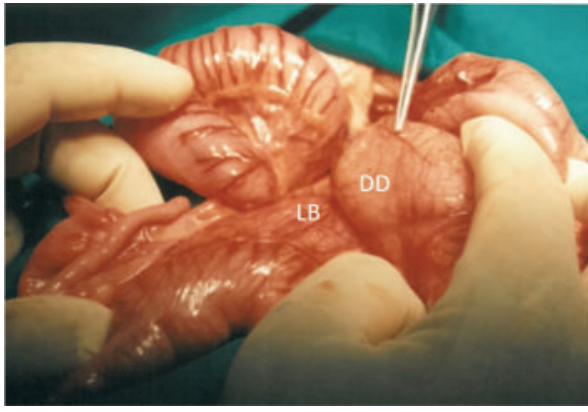

(b)

FIgURE 3: (a) Entry point of ileocolic intussusception (black arrow). (b) Ladd's bands (LB) with dilated duodenum (DD).

the superior mesenteric vessels; the ileocecal junction was freely mobile and the colon was suspended by primitive mesenteric folds. Furthermore, well-defined Ladd's bands were seen to extend from the ascending colon to the posterior abdominal wall across the duodenum (Figure 3(b)).

The intussusception was manually reduced, and no leading point was found. Ladd's procedure was also performed including appendicectomy. The child had an uneventful recovery. Six months after the operation he was well without any further abdominal symptoms and had gained weight.
The patient weighed $18 \mathrm{~kg}$ two weeks after the onset of symptoms and lost another $3 \mathrm{~kg}$ during the following four weeks. Six months after surgery, he weighed $22 \mathrm{~kg}$ (between 75th and 90th percentile).

\section{Discussion}

The incidence of chronic intussusception is about 3\% of all cases of intussusception in children aged under one year and approximately $10 \%$ of children over that age [7]. The true 


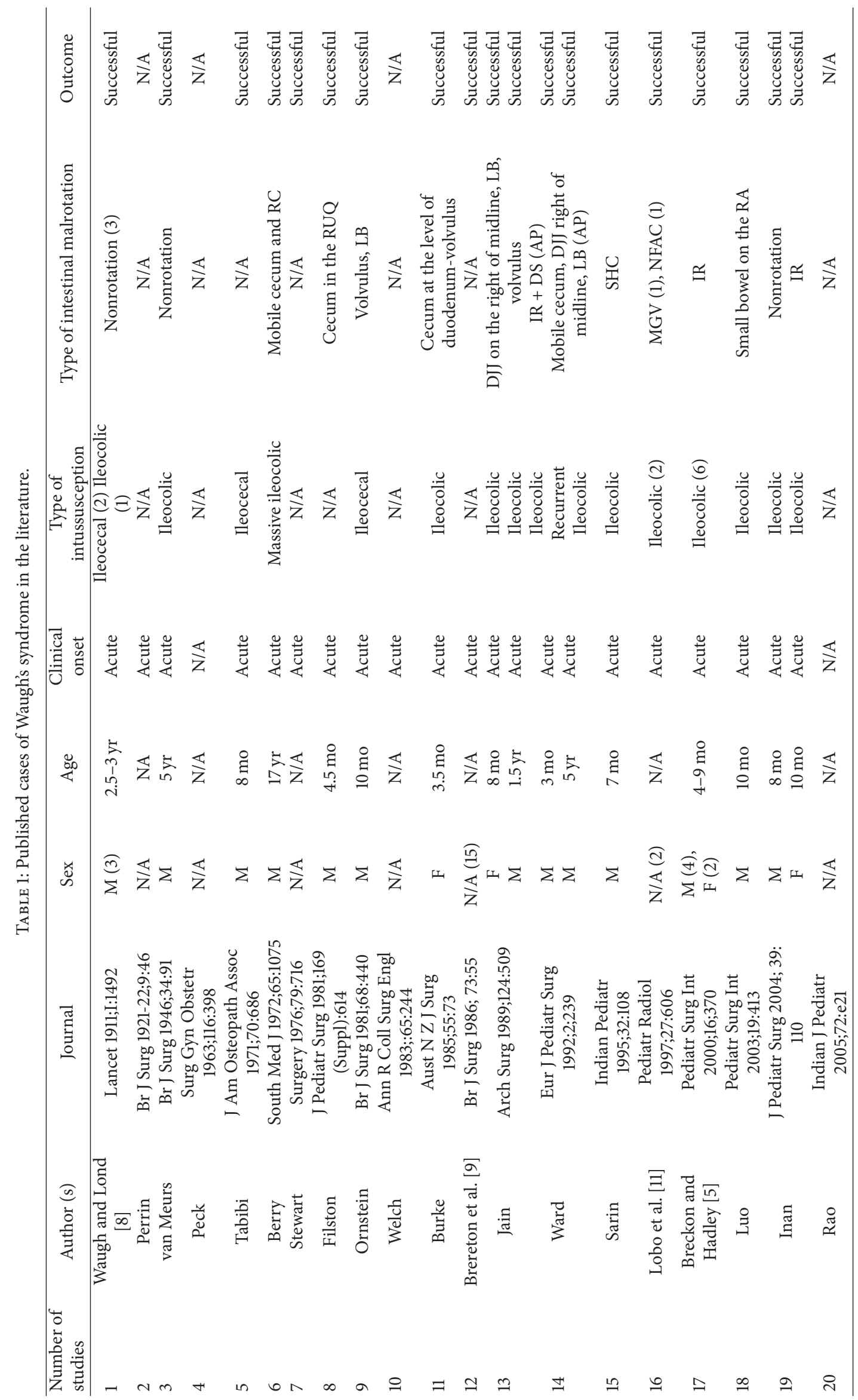




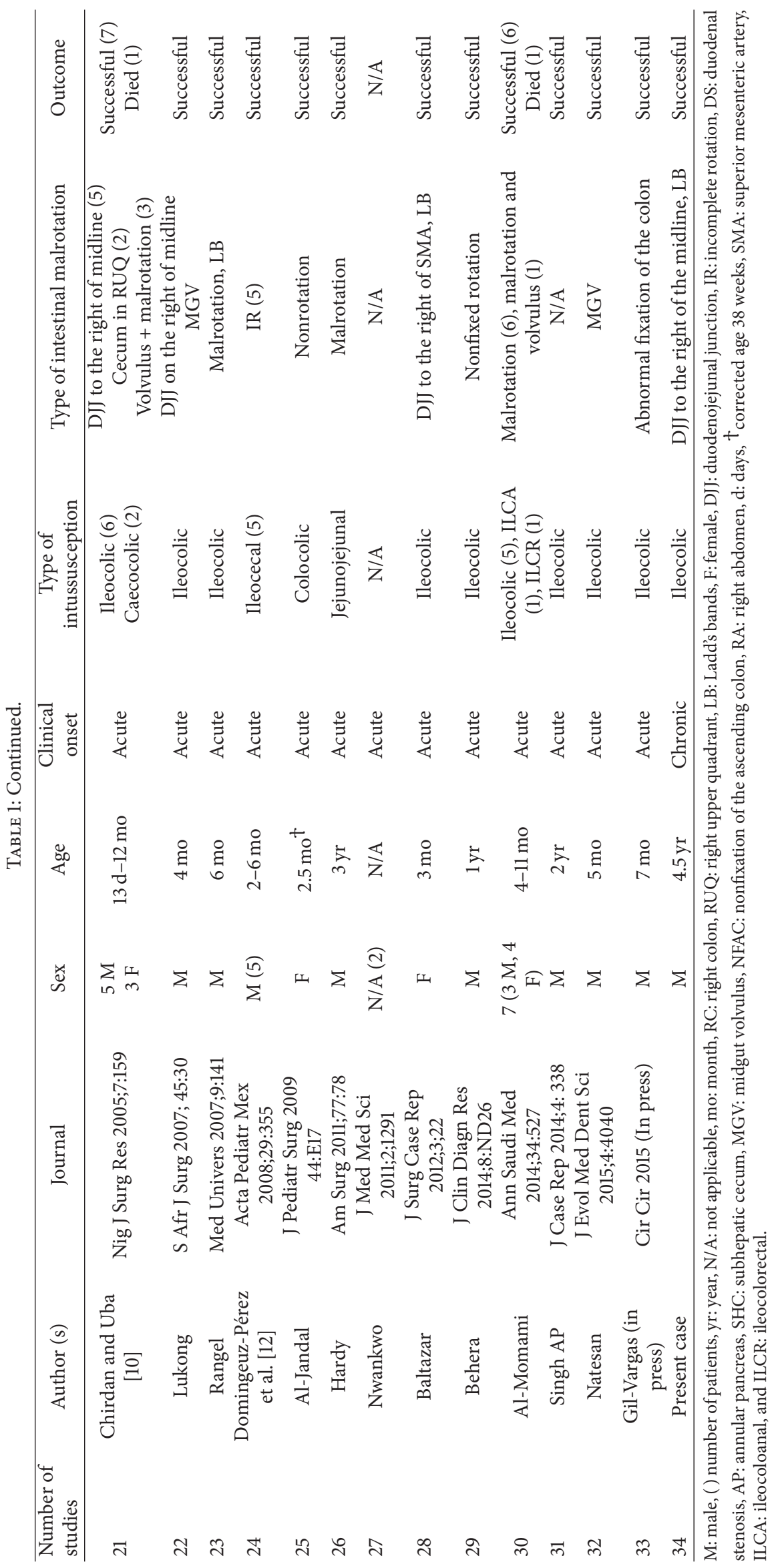


incidence of WS is not known [5]. A PubMed and Google Scholar search revealed 33 published studies that referred to 76 children with WS (age range, 13 days to 17 years) (Table 1). To our knowledge, the association of chronic intussusception with intestinal malrotation in children has never before been mentioned in the international literature and could represent a variant of WS.

Existing evidence suggests that intestinal malrotation may predispose to acute intussusception. Waugh and Lond originally suggested that an ascending and descending colon relatively unfixed to the posterior wall and freely suspended by its primitive mesenteric folds may provoke an ileocecal intussusception [8]. According to a study by Brereton et al. [9], the principal factor that allows the terminal ileum to pass into the cecum is abnormal fixation and rotation of the ileocecal mesentery, while Breckon and Hadley [5] suggested that a mobile right colon might predispose to intussusception. In our case, one could incriminate the possible role of the freely mobile ileocecal junction as a principal factor of chronic intussusception. Moreover, the primitive mesenteric folds which do not become sufficiently tense to occlude the mesenteric blood vessels may be the cause of longstanding recurrent abdominal symptoms without any further complications such as bowel necrosis.

Ultrasonography (U/S) and contrast enema both constitute reliable imaging tools in the diagnosis of acute intussusception [1]. In the case presented herein, U/S and barium contrast enema confirmed the diagnosis of chronic intussusception. That being said, radiological evaluation was seen to offer a definite preoperative diagnosis in only seven reports of the reviewed cases of WS (Table 1, studies 12, 16, 18, $23,25,29$, and 31).

In the majority of listed studies, open surgery (Table 1, studies 1-15, 17-22, 24, and 26-32) was the treatment of choice; a laparoscopic approach was performed in just one case (Table 1, study 25). Notably, in the studies by Breckon and Hadley [5] and Chirdan and Uba [10], intestinal malrotation was not taken into consideration during surgery for acute intussusception; hence, the patients were submitted to reoperation for recurrent symptoms of bilious vomiting. Furthermore, Lobo et al. [11] and DomingeuzPérez et al. [12] reported one and three cases, respectively, which were managed conservatively. In our case of a possible variant of WS, surgery was necessary to reduce the intussusception, given that the attempted hydrostatic reduction had failed. The operation had a successful outcome. All but two cases of the reviewed studies (Table 1, studies 21 and 29) were seen to have successful results.

\section{Conclusion}

The association between chronic intussusception and intestinal malrotation has never before been reported in the literature. This coexistence may represent a possible variant of WS. In cases of chronic intussusception, a high degree of suspicion is warranted in order to guide towards the proper diagnosis.

\section{Consent}

Informed and written consent for publication of this case report was obtained from the patient.

\section{Competing Interests}

The authors declare that there is no conflict of interests regarding the publication of this paper.

\section{References}

[1] B. T. Bucher, B. L. Hall, B. W. Warner, and M. S. Keller, "Intussusception in children: cost-effectiveness of ultrasound vs diagnostic contrast enema," Journal of Pediatric Surgery, vol. 46, no. 6, pp. 1099-1105, 2011.

[2] V. K. Pepper, A. B. Stanfill, and R. H. Pearl, "Diagnosis and management of pediatric appendicitis, intussusception, and Meckel diverticulum," Surgical Clinics of North America, vol. 92, no. 3, pp. 505-526, 2012.

[3] M. S. Shalaby, K. Kuti, and G. Walker, "Intestinal malrotation and volvulus in infants and children," British Medical Journal, vol. 347, Article ID f6949, 2013.

[4] N. Spigland, M. L. Brandt, and S. Yazbeck, "Malrotation presenting beyond the neonatal period," Journal of Pediatric Surgery, vol. 25, no. 11, pp. 1139-1142, 1990.

[5] V. M. Breckon and G. P. Hadley, "Waugh's syndrome: a report of six patients," Pediatric Surgery International, vol. 16, no. 5-6, pp. 370-373, 2000.

[6] B. I. Rees and J. Lari, "Chronic intussusception in children," British Journal of Surgery, vol. 63, no. 1, pp. 33-35, 1976.

[7] D. Macauley and T. Moore, "Subacute and chronic intussusception in infants and children," Archives Disease in Childhood, vol. 30, no. 150, pp. 180-183, 1955.

[8] G. E. Waugh and B. S. Lond, "Referred penile pain in intussusception with notes of three cases," The Lancet, vol. 177, no. 4579, pp. 1492-1494, 1911.

[9] R. J. Brereton, B. Taylor, and C. M. Hall, "Intussusception and intestinal malrotation in infants: Waugh's syndrome," British Journal of Surgery, vol. 73, no. 1, pp. 55-57, 1986.

[10] L. B. Chirdan and A. F. Uba, "Association of midgut malrotation with intussusception," Nigerian Journal of Surgical Research, vol. 7, no. 1-2, pp. 159-161, 2005.

[11] E. Lobo, A. Daneman, J. M. Fields, M. S. Keller, D. J. Alton, and B. Shandling, "The diagnosis of malrotation during air enema procedure," Pediatric Radiology, vol. 27, no. 7, pp. 606-608, 1997.

[12] S. T. Domingeuz-Pérez, C. Baeza-Herrera, G. Jaimes, M. L. Martinez-Rivera, and T. González-Mateos, "Sindrome de Waugh. Promeros informes en México," Acta Pediatrica Mexicana, vol. 29, no. 6, pp. 355-358, 2008. 


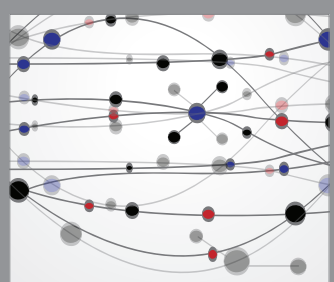

The Scientific World Journal
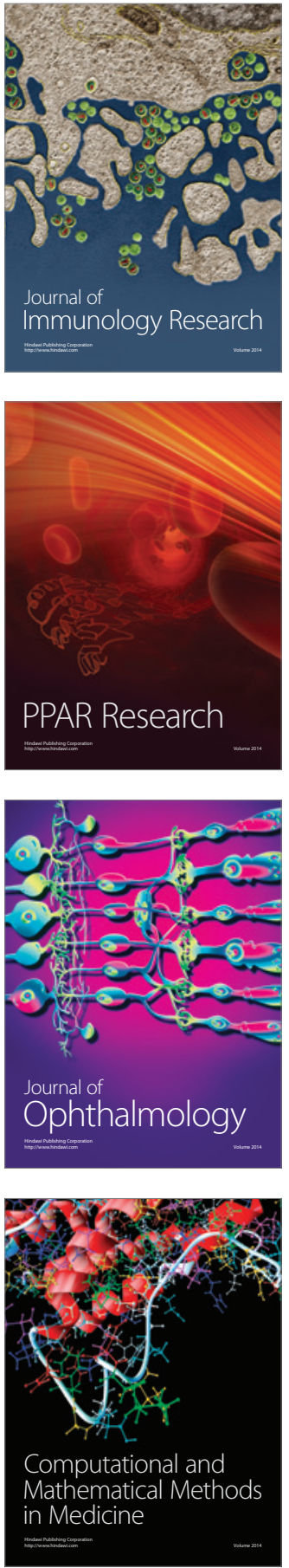

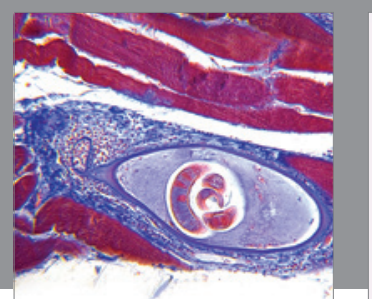

Gastroenterology Research and Practice

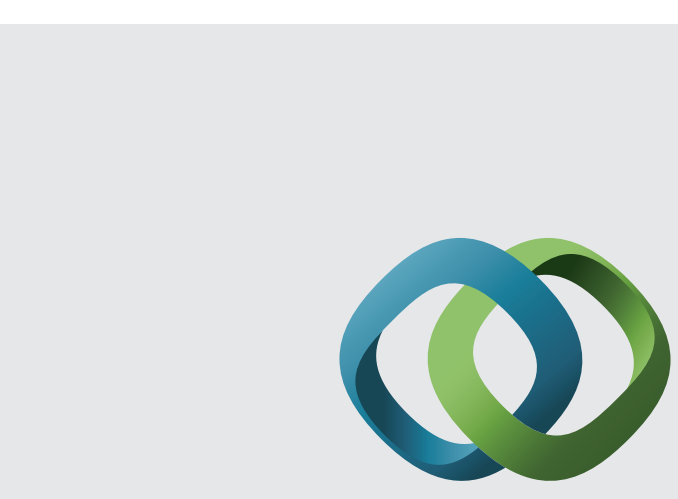

\section{Hindawi}

Submit your manuscripts at

http://www.hindawi.com
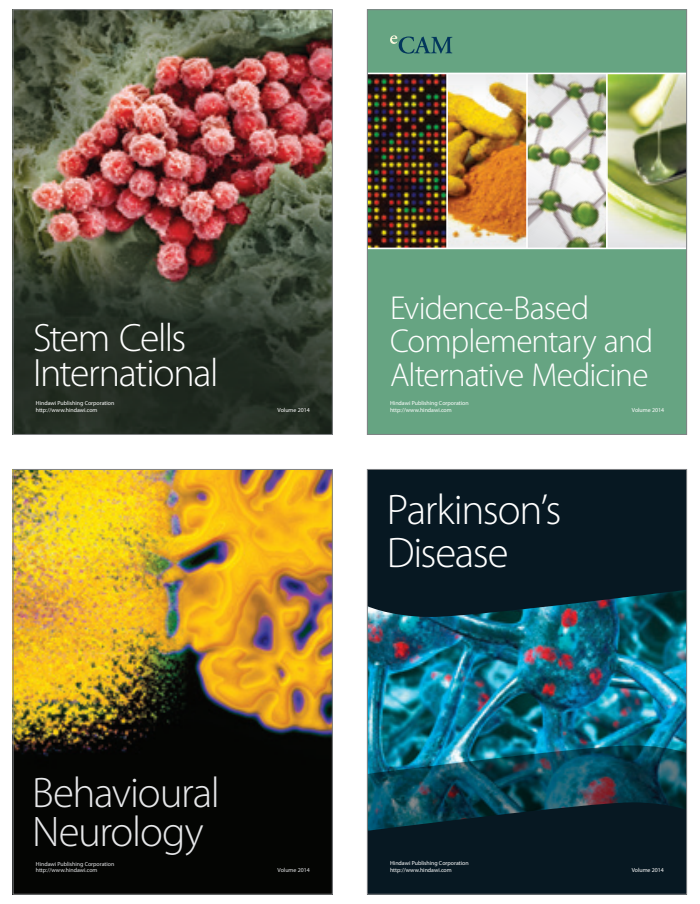
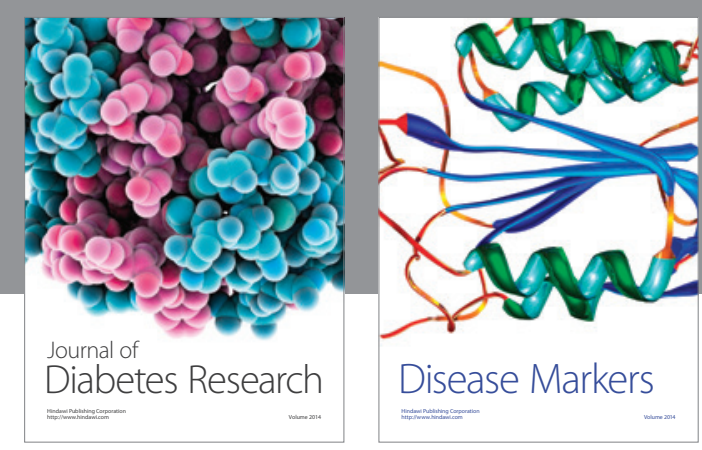

Disease Markers
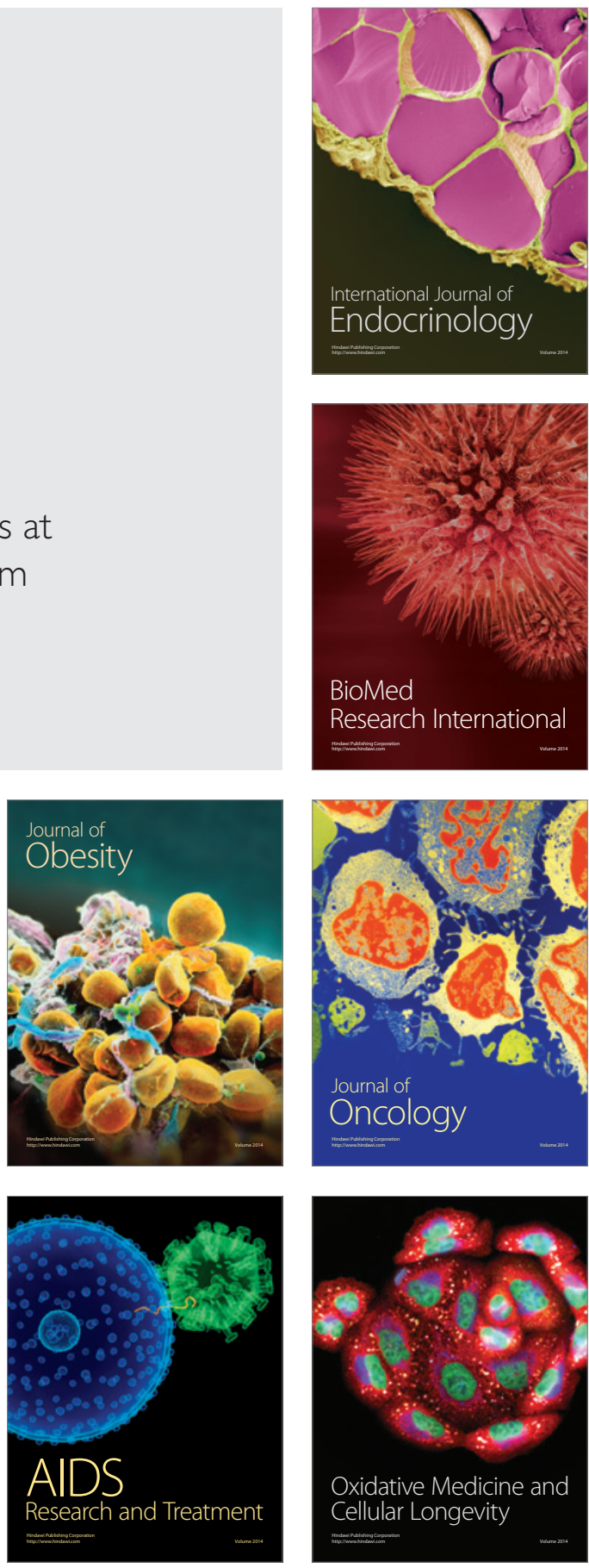